\title{
ROL DE LAS MANIFESTACIONES REUMÁTICAS Y DERMATOLÓGICAS EN EL DIAGNÓSTICO DE COVID-19 EN PACIENTES PAUCISINTOMÁTICOS
}

\author{
ROLE OF RHEUMATIC AND DERMATOLOGICAL MANIFESTATIONS IN THE \\ DIAGNOSIS OF COVID-19 IN PAUCIS-SYMPTOM PATIENTS
}

Urbano Solís ${ }^{(1)(2) @ ; ~ J o r g e ~ V a l d e ́ s ~}{ }^{(1)}$; Wilmar Hernández ${ }^{(3)}$; Ana Garofalo(4); Yarimi Rodríguez $^{(1)}$; Carlos Gafas ${ }^{(2)}$.

(1) Escuela Superior Politécnica de Chimborazo. Riobamba-Ecuador.

(2) Universidad Nacional de Chimborazo. Riobamba-Ecuador.

(3) Universidad de las Américas. Quito - Ecuador.

(4) Ministerio de Salud Pública del Ecuador.

Email:umsmwork74@gmail.com

https://doi.org/10.33789/talentos.7.2.137

\begin{abstract}
Resumen. La COVID-19 es una enfermedad sistémica que no solo incluye la presencia de manifestaciones respiratorias, sino también generales, neurológicas, cardiovasculares, dermatológicas y reumáticas. En muchas ocasiones este grupo de manifestaciones clínicas se presentan en ausencia de manifestaciones respiratorias. El objetivo de esta investigación fue identificar el rol de las manifestaciones dermatológicas y reumáticas como posible elemento facilitador del diagnóstico de COVID-19 en pacientes paucisintomáticos. Para esto se realizó una investigación básica, descriptiva y explicativa que incluyó a 111 pacientes con diagnóstico positivo de COVID-19. Los principales resultados incluyen la presencia de manifestaciones respiratorias en el 52,25\% de los casos, manifestaciones reumáticas en el 50,45\% y las dermatológicas en el 36,94\%. En pacientes sin manifestaciones respiratorias el 60,38\% presentó afectación reumática y el 45,28\% dermatológicas. Las lesiones maculopapulares $(60,96 \%)$, las mialgias (82,14\%) y las artralgias (62,59\%) fueron las manifestaciones
\end{abstract}

Recibido: 28 de septiembre de 2020 Aceptado: 17 de diciembre de 2020

Publicado como artículo cientifico en la Revista de Investigación Talentos VII (2), 74-83 
dermatológicas y reumáticas que con mayor frecuencia fueron identificadas en los pacientes con diagnóstico positivo de la enfermedad. Se concluye que existió una elevada frecuencia de presentación de manifestaciones reumáticas y dermatológicas en los pacientes con diagnóstico confirmado de COVID-19; resultado que se repitió dentro del grupo de pacientes confirmados de la enfermedad, pero sin presencia de manifestaciones respiratorias. La identificación de manifestaciones dermatológicas y reumáticas en pacientes sin expresión respiratoria puede constituir un elemento de sospecha clínica de COVID-19 en pacientes paucisintomáticos.

Palabras clave: COVID-19; Manifestaciones Dermatológicas; Manifestaciones Reumáticas; Pacientes paucisintomáticos.

Abstract. COVID-19 is a systemic disease that not only includes the presence of respiratory manifestations, but also general, neurological, cardiovascular, dermatological and rheumatic. On many occasions this group of clinical manifestations occur in the absence of respiratory manifestations. The objective of this research was to identify the role of dermatological and rheumatic manifestations as a possible facilitating element of the diagnosis of COVID-19 in paucisymptomatic patients. For this, a basic, descriptive and explanatory research was carried out that included 111 patients with a positive diagnosis of COVID-19. The main results include the presence of respiratory manifestations in $52.25 \%$ of cases, rheumatic manifestations in $50.45 \%$ and dermatological manifestations in $36.94 \%$. In patients without respiratory manifestations, $60.38 \%$ presented rheumatic involvement and $45.28 \%$ dermatological. Maculopapular lesions (60.96\%) and myalgias (82.14\%) and arthralgias (62.59\%) were the dermatological and rheumatic manifestations that were most frequently identified in patients with a positive diagnosis of the disease. It is concluded that there was a high frequency of presentation of rheumatic and dermatological manifestations in patients with a confirmed diagnosis of COVID-19; a result that was repeated within the group of patients confirmed with the disease but without the presence of manifestations. The identification of dermatological and rheumatic manifestations in patients without respiratory expression may constitute an element of clinical suspicion of COVID-19 in paucisymptomatic patients.

Keywords: COVID-19; Dermatological Manifestations; Rheumatic Manifestations; Paucisymptomatic patients.

\section{INTRODUCCIÓN}

La pandemia mundial conocida como COVID-19 ha concentrado la atención de los profesionales de la salud. El interés se justifica en sus principales características: rápida propagación, elevado número de contagios y muertes y la no existencia de esquemas terapéuticos y vacunas para controlar la enfermedad. Estos elementos centran los esfuerzos de investigación en aras de prevenir el contagio e identificar a los pacientes precozmente para minimizar la propagación de la enfermedad, sus complicaciones y la morbimortalidad que de la COVID-19 se genera (Solis Cartas, \& Martínez Larrarte, 
2020)

El elemento distinto de la COVID-19, en relación a las otras epidemias producidas por coronavirus, consiste en el elevado por ciento de pacientes paucisintomáticos, portadores del virus y sin manifestaciones respiratorias. Ante estos pacientes se ignora el padecimiento de la enfermedad y no se aplican medidas terapéuticas ni higiénico sanitarias, lo que facilita el contagio de la enfermedad y la aparición de complicaciones que ponen en peligro la vida de los pacientes.

Según datos ofrecidos por la Organización Mundial de la Salud, hasta el $80 \%$ de los pacientes con COVID-19 cursan sin manifestaciones respiratorias; alrededor del $14 \%$ de los pacientes cursan con manifestaciones respiratorias leves y el restante $6 \%$ desarrolla complicaciones respiratorias graves que pueden condicionar la muerte (Solis Cartas, 2020). Ante esta situación es necesario identificar posibles patrones clínicos que, ante la ausencia de manifestaciones respiratorias, puedan orientar hacia la sospecha clínica de la enfermedad.

En la actualidad se realizan investigaciones con el objetivo de identificar manifestaciones clínicas extrarespiratorias cuya presencia orienten al posible contagio con la enfermedad, incluso, en estadios iniciales, cuando aún no existe compromiso respiratorio. Este puede llegar a no presentarse nunca y entonces el paciente desconoce su condición de portador del virus y se comporta como una fuente de transmisión "asintomática" de la misma (Chia Proenza, Gómez Conde, \& del Toro Ravelo, 2020).
Dentro del grupo de manifestaciones extrarespiratorias de la COVID-19 se han descrito manifestaciones generales, neurológicas, cardiovasculares, reumáticas y dermatológicas (Gómez Conde, Chia Proenza, \& del Toro Ravelo, 2020). Estos dos últimos grupos tienen como característica común que pueden anteceder al cuadro respiratorio; por lo que la identificación de los patrones reumáticos y dermatológicos frecuentes de la COVID-19 pueden ser utilizados como un elemento de sospecha clínica temprana de la enfermedad (Tufan, \& Kayaaslan, 2020). Se describe que alrededor del 50\% de los pacientes con COVID-19 presentan manifestaciones reumáticas y dermatológicas como parte de la expresión sistémica de la enfermedad (Cai, Sun, \& Li, 2020).

Las manifestaciones reumáticas que con mayor frecuencia han sido asociadas a la COVID-19 son la fibromialgia, contracturas musculares, mialgias, artralgias y cuadros de artritis reactiva (Caso, et al, 2020) Los patrones de afectación dermatológica que son reportados en pacientes diagnosticados de la enfermedad son las lesiones acrales en forma de sabañones, erupciones vesiculosas, urticaria aguda, exantema maculopapular y las lesiones que orientan hacia la obstrucción vascular (livedo reticular, síndrome de Raynaud y otras) (Maqueda Zamora, et al, 2020).

Es por eso que, atendiendo al elevado número de contagios de la enfermedad, el porciento de pacientes paucisintomáticos y la elevada presencia de manifestaciones reumáticas y dermatológicos en estadios tempranos de la enfermedad y en pacientes paucisintomáticos; 
se decide realizar esta investigación con el objetivo de describir el rol que juegan las manifestaciones reumáticas y dermatológicas en el diagnóstico de la COVID-19 en pacientes paucisintomáticos.

\section{METODOLOGÍA}

Se realizó una investigación básica, no experimental, con un diseño descriptivo $\mathrm{y}$ explicativo y enfoque mixto al incluir elementos cualitativos y cuantitativos. El universo estuvo constituido por 119 pacientes con diagnóstico positivo de COVID-19. Para realizar el cálculo del tamaño de la muestra se utilizó la forma de cálculo muestral para poblaciones conocidas. Después de realizar los cálculos matemáticos se definió el total de la muestra en 111 pacientes. Se utilizó el método de muestreo aleatorio simple para conformar la muestra. Todos los pacientes incluidos en el estudio cumplieron los criterios de inclusión y exclusión definidos para la investigación.

Criterios de inclusión

- Pacientes con diagnóstico confirmado o de sospecha de COVID-19.

- Pacientes que expresaron, mediante la firma del consentimiento informado, su deseo de participar en la investigación.

Criterios de exclusión

- Pacientes con manifestaciones reumáticas o dermatológicas en los cuales no se confirmó la presencia de COVID-19.
- Pacientes que no estuvieron de acuerdo en firmar el consentimiento informado para formar parte de la investigación.

Se definieron las siguientes variables de investigación: presencia de manifestaciones reumáticas, tipo de manifestaciones reumáticas, presencia de manifestaciones dermatológicas, tipo de manifestaciones dermatológicas, presencia de manifestaciones respiratorias. Para el diagnóstico de la COVID-19 se tuvo en cuenta el resultado de la prueba de reacción en cadena a la polimerasa (PCR). Las manifestaciones reumáticas y dermatológicas fueron confirmadas por especialistas en reumatología y dermatología, facilitando la definición correcta de las mismas.

La observación, entrevista y la revisión documental fueron las técnicas de investigación utilizadas. La observación permitió identificar la presencia y tipo de manifestaciones reumáticas y dermatológicas. Además, permitió identificar si existieron manifestaciones respiratorias o no de la enfermedad. La entrevista consistió en una entrevista abierta a cada paciente con diagnóstico de la enfermedad que facilitó el conocimiento de características generales de los pacientes y otros elementos importantes relacionados con la presencia de las manifestaciones reumáticas y dermatológicas. Por su parte la revisión documental permitió consolidar conocimientos relacionados con la presencia de manifestaciones reumáticas y dermatológicas en pacientes con COVID-19 y la confirmación en la historia clínica de elementos generales relacionados con el 
diagnóstico y la evolución clínica de la enfermedad.

Toda la información recopilada fue homogenizada en una base de datos en Excel. El procesamiento de la información se realizó de forma automatizada mediante la utilización del programa estadístico SPSS en su versión 20,5 para Windows. Se determinaron medidas de tendencia central y de dispersión para las variables cuantitativas $\mathrm{y}$ frecuencias absolutas y porcentajes para las variables cualitativas. Se definió el nivel de confianza en el 95\%, el margen de error en el $5 \%$ y la significación estadística en una $\mathrm{p} \leq \mathrm{de}$ 0,05 . Todos los resultados fueron expresados en forma de tablas estadísticas para facilitar su comprensión.
Durante el desarrollo de la investigación se cumplieron las normas y procedimientos establecidos en la declaración de Helsinki II para la realización de investigaciones en seres humanos. La participación en el estudio se realizó de forma voluntaria, sin que generara gasto alguno para los pacientes y la incorporación de los mismos se realizó posterior a la firma del consentimiento informado. Los datos obtenidos fueron utilizados únicamente confines investigativos, no se utilizaron datos de identificación personal, solamente códigos alfa numéricos para establecer un orden a la información recopilada. La base de datos, con toda la información recopilada, fue destruida al terminar el informe final de la investigación.

\section{RESULTADOS Y DISCUSIÓN}

Tabla I. Distribución de pacientes con diagnóstico de COVID-19 según presencia de manifestaciones respiratorias, reumáticas y dermatológicas

\begin{tabular}{lcc}
\hline Presencia de manifestaciones & \multicolumn{2}{c}{ Muestra total $\mathbf{n = 1 1 1}$ pacientes } \\
& Frecuencia & Porciento \\
\hline Respiratorias & 58 & 52,25 \\
Reumáticas & 56 & 50,45 \\
Dermatológicas & 41 & 36,94 \\
Reumáticas y dermatológicas & 27 & 24,32 \\
\hline
\end{tabular}

Fuente: cuestionario de investigación

Se observa en la tabla 1 que solo el $52,25 \%$ de los pacientes con diagnóstico de COVID-19 presentaron manifestaciones respiratorias, por lo que el 47,75\% se comportó de forma paucisintomática sin presentar manifestaciones de tipo respiratoria. En relación a la presencia de manifestaciones reumáticas se puede observar que estuvieron presentes en el 50,45\% de los pacientes investigados y las manifestaciones dermatológicas en el 36,94\%. En el 24,32\% del total de casos positivos de COVID-19 incluidos en la investigación se presentaron tanto manifestaciones dermatológicas como reumáticas. 
Tabla II. Distribución de pacientes según tipo de manifestaciones reumáticas y dermatológicas identificadas.

\begin{tabular}{lccc}
\hline \multicolumn{1}{c}{ Tipo de manifestaciones } & $\begin{array}{c}\text { Frecuencia } \\
\text { Dermatológicas } \mathbf{n = 4 1}\end{array}$ & $\begin{array}{c}\text { Porciento } \\
\text { pacientes }\end{array}$ & $\mathbf{p}$ \\
Lesiones acrales & 14 & 34,15 & 0,066 \\
Maculopápulas & 25 & 60,96 & 0,04 \\
Urticaria aguda & 21 & 51,22 & 0,049 \\
Erupción vesicular & 17 & 41,46 & 0,059 \\
Livedo reticular & 13 & 32,74 & 0,068 \\
Síndrome de Raynaud & 7 & 17,07 & 0,083 \\
& Reumáticas $\mathbf{n = 5 6}$ pacientes & \\
Mialgias & 46 & 82,14 & 0,018 \\
Contractura muscular & 16 & 28,57 & 0,072 \\
Fibromialgia & 24 & 42,86 & 0,057 \\
Artralgia & 35 & 62,50 & 0,038 \\
Artritis reactiva & 19 & 33,93 & 0,067 \\
Síndrome oligoarticular & 9 & 16,07 & 0,084 \\
\hline Fuente: cuestionario de investigación & $\mathbf{p} \leq \mathbf{0 , 0 5}$ & &
\end{tabular}

Se muestra como datos significativos la las de mayor porciento de presentación y presencia del $60,96 \%$ de pacientes con maculopápulas $(\mathrm{p}=0,04)$ y del $51,22 \%$ significación estadística fueron las mialgias $(82,14 \%$ y $p=0,018)$ y las artralgias $(62,5 \%$ de casos con urticaria aguda $(p=0,049)$. Existieron otras manifestaciones dermatológicas identificadas como fueron las erupciones vesiculares (41,46\%), lesiones acrales $(34,15 \%)$, livedo reticular $(32,74 \%)$ y el síndrome de Raynaud (17,07\%). En relación a las manifestaciones reumáticas y $\mathrm{p}=0,038)$. También estuvieron presentes, aunque no aportaron significación estadística, la fibromialgia $(42,86 \%)$, la artritis reactiva $(33,93 \%)$, las contracturas musculares $(28,57 \%)$ y la presencia de síndrome de Raynaud que se identificó en el 16,07\% de casos investigados (tabla 2).

Tabla III. Distribución de pacientes con diagnóstico de COVID-19, según presencia de manifestaciones dermatológicas en ausencia de manifestaciones respiratorias.

\begin{tabular}{lccc}
\hline $\begin{array}{l}\text { Presencia y tipo de manifesta- } \\
\text { ciones dermatológicas }\end{array}$ & \multicolumn{2}{c}{$\begin{array}{c}\text { Muestra total de 53 pacientes con COVID-19 sin manifestaciones } \\
\text { respiratorias. }\end{array}$} \\
\cline { 2 - 4 } & Frecuencia & Porciento & $\mathbf{p}$ \\
Presencia & $\mathbf{2 4}$ & $\mathbf{4 5 , 2 8}$ & -- \\
Ausencia & $\mathbf{2 9}$ & $\mathbf{5 4 , 7 2}$ & -- \\
\hline Tipo de manifestaciones dermatológicas $\mathbf{n}=\mathbf{2 4}$ pacientes & & \\
Lesiones acrales & 4 & 16,67 & 0,083 \\
Maculopápulas & 9 & 37,50 & 0,063 \\
Urticaria aguda & 7 & 29,17 & 0,07 \\
Erupción vesicular & 4 & 16,67 & 0,083 \\
Livedo reticular & 6 & 25,00 & 0,075 \\
Síndrome de Raynaud & 3 & 12,50 & 0,088 \\
\hline Fuente: cuestionario de investigación & $\mathbf{p} \leq \mathbf{0 , 0 5}$ &
\end{tabular}


Al analizar la presencia y comportamiento de las manifestaciones dermatológicas en los pacientes con COVID-19 paucisintomáticos se obtuvo que las manifestaciones dermatológicas se presentaron hasta en el $45,28 \%$ de este tipo de pacientes. Dentro de ellas las lesiones maculopapulares (37,50\%), los cuadros de urticaria aguda $(29,17 \%)$ y el livedo reticular $(25,00 \%)$ fueron las de mayor frecuencia y porciento de presentación. Ninguna de ellas presentó significación estadística.

Tabla IV. Distribución de pacientes con diagnóstico de COVID-19, según presencia de manifestaciones reumáticas en ausencia de manifestaciones respiratorias.

\begin{tabular}{|c|c|c|c|}
\hline \multirow[t]{2}{*}{$\begin{array}{l}\text { Presencia y tipo de mani- } \\
\text { festaciones reumáticas }\end{array}$} & \multicolumn{3}{|c|}{$\begin{array}{l}\begin{array}{l}\text { Muestra total de } 53 \text { pacientes con COVID-19 sin manifestaciones respirato- } \\
\text { rias. }\end{array} \\
\end{array}$} \\
\hline & Frecuencia & Porciento & $\mathbf{p}$ \\
\hline Presencia & 32 & 60,38 & ---- \\
\hline Ausencia & 21 & 39,62 & ---- \\
\hline \multicolumn{4}{|c|}{ Tipo de manifestaciones reumáticas $n=32$ pacientes } \\
\hline Mialgias & 21 & 65,62 & 0,034 \\
\hline Contractura muscular & 11 & 34,38 & 0,066 \\
\hline Fibromialgia & 9 & 28,12 & 0,072 \\
\hline Artralgia & 18 & 56,25 & 0,044 \\
\hline Artritis reactiva & 16 & 50,0 & 0,05 \\
\hline Síndrome oligoarticular & 3 & 9,38 & 0,091 \\
\hline
\end{tabular}

Se puede apreciar en la tabla 4 que el $60,38 \%$ de los pacientes con diagnóstico de COVID-19 que no presentaron manifestaciones respiratorias sí presentaron manifestaciones reumáticas. Dentro de estas destacaron la presencia de mialgias $(65,62 \%$ y $\mathrm{p}=0,064)$, artralgias $(56,25 \%$ y $\mathrm{p}=0,044)$ $\mathrm{y}$ las artritis reactivas $(50,0 \%$ y $\mathrm{p}=0,05)$; todas presentaron resultados significativos. Otras manifestaciones reumáticas que se presentaron en pacientes sin manifestaciones respiratorias fueron las contracturas musculares $(34,38 \%)$, fibromialgia $(28,12 \%)$ y el síndrome oligoarticular $(9,38 \%)$.

\section{Discusión}

Como ha sido descrito con anterioridad, distintos estudios destacan el elevado porcentaje de pacientes paucisintomáticos entre los casos con diagnóstico de COVID-19. La ausencia de manifestaciones respiratorias constituye un elemento favorecedor del contagio de la enfermedad, propiciando una mayor propagación de la misma (Góngora Gómez, \& Gámez Leyva, 2020).

Las manifestaciones reumáticas y dermatológicas estuvieron presentes en un importante por ciento de pacientes diagnosticados, incluso dentro del grupo de pacientes paucisintomáticos que no presentaban manifestaciones respiratorias. Este resultado es similar al de otras investigaciones que reportan que dentro de las manifestaciones extra respiratorias de la COVID-19 las reumáticas y dermatológicas están presentes con elevada frecuencia (Granados Campos, et al, 2020). 
Este resultado evidencia la importancia que revista la identificación de manifestaciones reumáticas y dermatológicas como posibles patrones de sospecha clínica de la COVID-19 que posteriormente debe confirmarse con estudios de laboratorio que demuestren la viremia. En esta investigación, al igual que otros reportes(Macías, etal, 2020), las lesiones maculopapulares fueron la manifestación dermatológica que con mayor frecuencia se identificó entre el total de pacientes confirmados de la enfermedad y dentro de aquellos que no presentaban manifestaciones respiratorias dentro del cortejo sintomático de la COVID-19. Este último resultado no fue posible comparar con otros reportes ya que no se pudieron identificar investigaciones que abordaran este mismo tema.

En el caso de las manifestaciones reumáticas existió un comportamiento similar tanto en la totalidad de los pacientes como en el análisis de la presencia de las mismas en los pacientes con diagnóstico positivo de COVID-19 pero sin expresión respiratoria de la enfermedad. En el primero de los casos, las mialgias, artralgias, la fibromialgia y las artritis reactivas fueron las de mayor frecuencia de aparición. En el grupo de pacientes sin manifestaciones respiratorias, las mialgias, artralgias y la artritis reactiva fueron de la mayor frecuencia de identificación. Este dato tampoco pudo ser comparado con otras investigaciones ya que no se identificaron estudios que reportaran este tipo de resultado.

A pesar de la limitación de comparación de algunos de los resultados de esta investigación, su principal aporte desde el punto de vista científico se relaciona directamente con la evidencia de la elevada presencia de manifestaciones dermatológicas y reumáticas en los pacientes con COVID-19 confirmados. Más importante aún es cómo se mantienen los porcentajes de presentación de este tipo de manifestaciones en los pacientes con diagnóstico de la enfermedad, pero sin expresión respiratoria de la misma. A partir de este resultado se puede plantear la hipótesis de que la identificación de manifestaciones reumáticas y dermatológicas puede constituir el elemento de sospecha clínica necesario para identificar pacientes portadores de COVID-19 sin manifestaciones respiratorias.

De comprobarse la veracidad de esta hipótesis constituirá, quizás, el resultado más adelantado y esperanzador en pos de detener el contagio de la enfermedad.

Se concluye que existió una elevada frecuencia de presentación de manifestaciones reumáticas y dermatológicas en los pacientes con diagnóstico confirmado de COVID-19; resultado que se repitió dentro del grupo de pacientes confirmados de la enfermedad, pero sin presencia de manifestaciones. La identificación de manifestaciones dermatológicas y reumáticas en pacientes sin expresión respiratoria puede constituir un elemento de sospecha clínica de COVID-19 en pacientes paucisintomáticos.

\section{REFERENCIAS BIBLIOGRÁFICAS}

Cai, S., Sun, W., \& Li, M. (2020). A complex COVID-19 case with rheumatoid arthritis treated with tocilizumab. Clin Rheumatol, 39, 2797-280. Recuperado de https://doi. org/10.1007/s10067-020-05234-W 
Caso, F., Costa, L., Ruscitti, P., Navarini, L., Del Puente, A., \& Giacomelli, R. (2020). Could Sars-coronavirus-2 trigger autoimmune and/or autoinflammatory mechanisms in genetically predisposed subjects? Autoimmunity Reviews, 19(5)102524. Recuperado de: https://www. sciencedirect.com/science/article/pii/ S1568997220300781

Chia Proenza, D., Gómez Conde, S.Y., \& del Toro Ravelo, L.M. (2020). Acercamiento a la COVID-19 desde una perspectiva pediátrica. Rev Cubana de Reumatolo, 22(2). Recuperado de http://www. revreumatologia.sld.cu/index.php/ reumatologia/article/view/830

Gómez Conde, S.Y., Chia Proenza, D., \& del Toro Ravelo, L.M. (2020). Revisión de las recomendaciones para enfermos reumáticos y la COVID-19. Propuesta para los pacientes pediátricos cubanos con enfermedad reumática y sus familiares. Rev Cubana de Reumatolo,22(2),118-124. Recuperado de: http://www.revreumatologia.sld. cu/index.php/reumatologia/article/ view/831

Góngora Gómez, O., \& Gámez Leyva, L.R. (2020). Manifestaciones extrapulmonares de la infección por el nuevo Coronavirus SARSCoV-2. Rev haban cienc méd, 19(Suppl 1),e3378. Recuperado de: http://scielo.sld.cu/scielo. php? script=sci arttext\&pid=S1729-

\section{$\underline{519 \times 2020000400006 \& \operatorname{lng}=\mathrm{es}}$}

Granados Campos, L., Broche del Pino, L., Pérez Leal, L., \& López Rodríguez, V.M. (2020). Manifestaciones cutáneas en pacientes pediátricos infectados por el coronavirus SARS-CoV-2. Rev Cubana Pediatr,92(Supp11), e1171. Recuperado de:

http://scielo.sld.cu/scielo. php?script $=$ sci_arttext\&pid=S0034$75312020000500009 \& \operatorname{lng}=\mathrm{es}$

Macías, Ma.A., Zapata, E., González, Ma.C., Fuenzalida, H., Honeyman, J., Chávez, F., et al. Recomendaciones Rama de Dermatología Pediátrica, Sociedad Chilena de Pediatría. Pandemia COVID-19. Rev. chil. pediatr., 24(3), 109-135. Recuperado de: https://scielo.conicyt.cl/scielo. php?script=sci arttext\&pid=S0370$\underline{41062020005001005 \& \operatorname{lng}=\mathrm{es}}$

Maqueda Zamora, G., Sierra Santos, L., Sierra Santos, E., Martínez Ballester, J.F. (2020). Manifestaciones dermatológicas de la infección por COVID-19 en Pediatría. Rev Clin Med Fam,13(2),166-70. Recuperado de: http://scielo.isciii.es/scielo. php? script=sci_arttext\&pid=S1699$\underline{695 X 2020000200166 \& \operatorname{lng}=\mathrm{es}}$

Solis Cartas, U., \& Martínez Larrarte, J. (2020). Opciones terapéuticas al síndrome de liberación de citocinas en pacientes con la COVID-19. Revista Cubana de Medicina Militar, 49(3), e0200783. Recuperado de: http://www. 
revmedmilitar.sld.cu/index.php/mil/

article/view/783

Solis Cartas, U. (2020). Coronavirus

y enfermedades reumáticas,

suposiciones, mitos y realidades.

Rev Cubana de Reumatolo,22(2).

Recuperado de: http://revreumatologia.

sld.cu/index.php/reumatologia/article/

view/791

Tufan, Z.K., \& Kayaaslan, B. (2020).

Crushing the curve, the role of

national and international institutions

and policy makers in COVID-19

pandemic. Turk J Med Sci.,

50(SI-1),495- 508. 